\title{
Cell intrinsic regulation of external hematopoietic stem cell stress
}

\author{
Frank J. T. Staal \\ Department of Immunohematology and Blood Transfusion, Leiden University Medical Center, Leiden, The Netherlands \\ Correspondence to: Prof. Frank J. T. Staal. Department of Immunohematology and Blood Transfusion, Leiden University Medical Center, Albinusdreef \\ 2, building 1, L3-35, P.O. Box 9600, 2300 RC Leiden, The Netherlands. Email: F.J.T.Staal@lumc.nl. \\ Provenance: This is an invited Editorial commissioned by the Editor-in-Chief Zhizhuang Joe Zhao (Pathology Graduate Program, University of \\ Oklahoma Health Sciences Center, Oklahoma City, USA). \\ Comment on: Kleppe M, Spitzer MH, Li S, et al. Jak1 integrates cytokine sensing to regulate hematopoietic stem cell function and stress \\ hematopoiesis. Cell Stem Cell 2018;22:277.
}

Received: 05 April 2018; Accepted: 19 April 2018; Published: 22 May 2018.

doi: $10.21037 /$ sci.2018.05.01

View this article at: http://dx.doi.org/10.21037/sci.2018.05.01

Hematopoietic stem cells (HSCs) have the ability to self-renew and generate all lineages of blood cells. Most HSCs are resting in adult life and the balance between differentiation, self-renewal, quiescence and apoptosis is strictly regulated (1). Hence, a wide variety of interconnecting signals operates on HSCs. Most of these are found in a specialized microenvironment, the stem cell niche $(2,3)$, where HSCs are localized and protected from direct stressors. Such signals include conserved evolutionary pathways like the Wnt $(4,5)$, Notch (6) and bone morphogenic proteins (BMP) (7) cascades, but also hematopoietic cytokines, such as stem cell factor (SCF) and thrombopoietin (TPO) (8). However, under conditions where a large supply of blood cells are needed, certain inflammatory cytokines can provide signals that induce rapid proliferation and differentiation of HSCs (9). These signals mainly concern cytokines such as interferon (IFN) $-\alpha / \beta$ and IFN- $\gamma$ as well as granulocyte colonystimulating factor (G-CSF) and granulocyte-macrophage colony-stimulating factor (GM-CSF). Interestingly, some of these signals are used clinically to "mobilize" stem cells to the peripheral blood for harvesting stem cells for transplantation purposes (10). The combined laboratories of Ross Levine and Garry Nolan have now better investigated how the common signaling molecules underneath these inflammatory cytokine-receptors regulate HSC stem cell biology under stress (11).

Type I interferons (IFN- $\alpha$ and $-\beta$ ) are secreted cytokines that are produced by a variety of immune cells in response to all kind of infections or through the recognition of tumor cells. IFN- $\alpha$ and IFN- $\beta$ signal via a receptor consisting of interferon $\alpha$ receptors 1 and 2 (IFNAR1 and IFNAR2). IFN- $\gamma$ signals via a receptor composed of IFNGR1 and IFNGR2 $(12,13)$. IFN- $\gamma$ plays a critical role in innate immunity to intracellular pathogens and also activates macrophages and Th1 CD4 $\mathrm{T}$ cell responses.

IFNs play a critical role in the regulation of resistance to viral infection and activation of the innate and acquired immune system through IFN-stimulated genes. IFNs also have an anti-tumor effect as the IFN regulated target genes not only inhibit viral replication and clear infected cells but can also help in attacking cancer cells. To induce the expression of IFN-target genes, IFN signaling is mediated by activation of the JAK-STAT signaling pathway through the type I IFN receptor [reviewed in $(12,13)$ ].

Because of their ability to regulate immune responses, recombinant type I interferons have been used clinically to treat viral infections, solid tumors and hematological malignancies, including multiple myeloma. Interestingly, while on most target cells IFNs have an anti-proliferative effect (growth arrest), they have an opposite effect on stem cells. Two seminal articles by Essers et al. (14) and Sato et al. (15) have reported that IFNs can induce HSC to proliferate. Essers et al. found that high levels of IFNa induced HSC proliferation; these IFN- $\alpha$-stimulated HSCs are functionally compromised as they are outcompeted by non-activated HSCs in repopulation assays (14). Sato et al. found that mice lacking a component of the IFN signaling pathway (IRF2) had an unexpected escape from quiescence within the HSC pool. These investigators studied mice 
that were genetically deficient for a negative regulator of type I interferon signaling, interferon response factor 2 [IRF2 (15)]. HSCs in these mice show chronic proliferation leading to a profound stem cell defect in secondary transplantations. Blocking IFN signaling in $\mathrm{IRF}^{-/-} \mathrm{HSCs}$, however restored their ability to repopulate irradiated recipient mice (15). This observation suggests that IRF2 normally suppresses IFN signaling in wild type HSCs, maintaining these cells mostly in a dormant state (which under homeostasis is the case for $>95 \%$ of HSCs)

The very different effects of IFNs on different types of target cells, has prompted much research into the signaling pathways emanating from their receptors. Clearly the JAK tyrosine kinases are critical mediators of these effects and hence targeted mutation of these receptors has been subject of several important publications, including a recent one by Kleppe et al. (11) who used conditional floxed Jak1 knockout mice in which deletion has been induced using two different Cre promoters (Mx-Cre and ERT-Cre). Loss of Jak1 in HSCs resulted in a severe decrease in longterm repopulating HSCs, while an increase in myeloid progenitors was observed. Consistently, Jak1 deficient HSCs showed 3-5 folds lower reconstitution ability in competitive translation assays when tested against Cre-negative control HSCs. Also, in stress hematopoiesis induced by $5-\mathrm{FU}$, these HSCs showed marked defects. Authors showed that not only cytokine responses to interferons were reduced, but also to interleukin-3 (IL-3), while IL-6 responses were intact. The latter result may explain the puzzling observation that while IFN- $\alpha$ activated HSCs show poor reconstitution, IFN- $\alpha$ receptor $^{-/-}$HSC do not show any defect. Jak1 is the first downstream signaling component and integrates signals not only from IFN- $\alpha$ but also other cytokines, notably IL-3.

Moreover, Kleppe et al. addressed possible redundancy between Jak1 and other Janus kinases (Jak1 is part of a four-member family of kinases, Jak1/2/3 and Tyk2) by using a constitutively activated Jak2 allele (which also can cause leukemia), as provided by the Jak2V617F knock-in mice (16). Mice lacking Jak1, but having the Jak2 activated allele showed some restoration of HSC function, but not to wildtype levels and certainly not to the level of Jak2 gain of function mutants in a wildtype background. Hence, there is only partial functional redundancy between these two related kinases in vivo.

The straight Jak1 knock-out mice die around birth (17) and fetal liver HSC transplantations have mainly shown lack of IL7 responses resulting in reduced numbers of thymocytes, pre- $\mathrm{B}$ cells, and mature $\mathrm{T}$ and $\mathrm{B}$ lymphocytes but no other defects. Hence the conditional KO, which has been used before to explore B lymphopoiesis and NK cell development (18), were very useful to investigate HSC function.

Jak1 and Jak2 gain of function mutations are found in human hematological malignancies $(19,20)$. Jak inhibitors are therefore used clinically to treat various types of leukemia and myeloproliferative neoplasms (MNPs). However, as these studies highlight, unexpected side effects from long term profound Jak1 inhibition may occur and could even result in stem cell failure if these results in mice were extrapolated to humans. Most likely, dosage of such drugs and combination therapy targeting other pathways are important to maximize efficacy in treating the malignancy while minimizing such dramatic possible side effects.

\section{Acknowledgements}

Funding: Frank J. T. Staal is supported in part by grants from ZonMW (40-41900-98-020), EU H2020 SCIDNET and EU H2020 RECOMB.

\section{Footnote}

Conflicts of Interest: The author has no conflicts of interest to declare.

\section{References}

1. Luis TC, Killmann NM, Staal FJ. Signal transduction pathways regulating hematopoietic stem cell biology: introduction to a series of Spotlight Reviews. Leukemia 2012;26:86-90.

2. Lo Celso C, Klein RJ, Scadden DT. Analysis of the hematopoietic stem cell niche. Curr Protoc Stem Cell Biol 2007; Chapter 2:Unit 2A.5.

3. Wilson A, Trumpp A. Bone-marrow haematopoietic-stemcell niches. Nat Rev Immunol 2006;6:93-106.

4. Luis TC, Ichii M, Brugman MH, et al. Wnt signaling strength regulates normal hematopoiesis and its deregulation is involved in leukemia development. Leukemia 2012;26:414-21.

5. Luis TC, Naber BA, Roozen PP, et al. Canonical wnt signaling regulates hematopoiesis in a dosage-dependent fashion. Cell Stem Cell 2011;9:345-56.

6. Pajcini KV, Speck NA, Pear WS. Notch signaling in mammalian hematopoietic stem cells. Leukemia 
2011;25:1525-32.

7. Singbrant S, Karlsson G, Ehinger M, et al. Canonical BMP signaling is dispensable for hematopoietic stem cell function in both adult and fetal liver hematopoiesis, but essential to preserve colon architecture. Blood 2010;115:4689-98.

8. Blank U, Karlsson G, Karlsson S. Signaling pathways governing stem-cell fate. Blood 2008;111:492-503.

9. Trumpp A, Essers M, Wilson A. Awakening dormant haematopoietic stem cells. Nat Rev Immunol 2010;10:201-9.

10. Hoggatt J, Pelus LM. Many mechanisms mediating mobilization: an alliterative review. Curr Opin Hematol 2011;18:231-8.

11. Kleppe M, Spitzer MH, Li S, et al. Jak1 Integrates Cytokine Sensing to Regulate Hematopoietic Stem Cell Function and Stress Hematopoiesis. Cell Stem Cell 2018;22:277.

12. Ihle JN, Kerr IM. Jaks and Stats in signaling by the cytokine receptor superfamily. Trends Genet 1995;11:69-74.

13. Ihle JN, Nosaka T, Thierfelder W, et al. Jaks and Stats in cytokine signaling. Stem Cells 1997;15 Suppl 1:105-11; discussion 112 .

doi: $10.21037 /$ sci.2018.05.01

Cite this article as: Staal FJ. Cell intrinsic regulation of external hematopoietic stem cell stress. Stem Cell Investig 2018;5:16.
14. Essers MA, Offner S, Blanco-Bose WE, et al. IFNalpha activates dormant haematopoietic stem cells in vivo. Nature 2009;458:904-8.

15. Sato T, Onai N, Yoshihara H, et al. Interferon regulatory factor-2 protects quiescent hematopoietic stem cells from type I interferon-dependent exhaustion. Nat Med 2009;15:696-700.

16. Mullally A, Lane SW, Ball B, et al. Physiological Jak2V617F expression causes a lethal myeloproliferative neoplasm with differential effects on hematopoietic stem and progenitor cells. Cancer Cell 2010;17:584-96.

17. Rodig SJ, Meraz MA, White JM, et al. Disruption of the Jak1 gene demonstrates obligatory and nonredundant roles of the Jaks in cytokine-induced biologic responses. Cell 1998;93:373-83.

18. Blasius AL, Barchet W, Cella M, et al. Development and function of murine B220+CD11c+NK1.1+ cells identify them as a subset of NK cells. J Exp Med 2007;204:2561-8.

19. Koppikar P, Bhagwat N, Kilpivaara O, et al. Heterodimeric JAK-STAT activation as a mechanism of persistence to JAK2 inhibitor therapy. Nature 2012;489:155-9.

20. Meyer SC, Levine RL. Molecular pathways: molecular basis for sensitivity and resistance to JAK kinase inhibitors. Clin Cancer Res 2014;20:2051-9. 